\title{
2017 Manuscript Reviewers
}

The following individuals generously offered their time and expertise as manuscript reviewers on one or more occasions between 23 November 2016 and 18 November 2017. We are very grateful for their assistance.

\begin{tabular}{|c|c|c|c|}
\hline Aars, J. & Dowsley, M.K. & Kellett, D.K. & Schmutz, J. \\
\hline Adamczewski, J. & Druckenmiller, M. & Kitson, $\mathrm{P}$. & Schott, S. \\
\hline Andres, B.A. & Dumond, D.E. & Kofinas, G. & Smith, T.S. \\
\hline Arp, C. & Dumond, M. & Koponen, S. & Smol, J.P. \\
\hline Asmus, A. & Durner, G.M. & Koski, W.R. & Southcott, C. \\
\hline Balasse, M. & Evans, M. & Küpper, F.C. & Stafford, K.M. \\
\hline Behe, C. & Exner-Pirot, H. & Lajeunesse, A. & Stephenson, S. \\
\hline Benkert, B. & Farré, A.B. & Larsson, K. & Stewart, E.J. \\
\hline Biersma, E.M. & Festa, J. & Lindstrom, $\mathrm{S}$. & Stewart, R.E.A. \\
\hline Bishop, S.C. & Fidel, M. & Lyons, E.A. & Taylor, G.M. \\
\hline Blackwell, S.B. & Flood, P. & MacLaren, I.S. & Thiemann, G.W. \\
\hline Boccaletti, C. & Fulford, T. & Manandhar, S. & Thienpont, J. \\
\hline Borchers, D. & Gallagher, C.P. & Melling, $\mathrm{H}$. & Trukhanova, I.S. \\
\hline Boudreau, S. & Galley, R.J. & Merrill, E. & Turner, C. \\
\hline Boveng, P. & Galloway, T. & Mochnacz, N.J. & Van der Jeugd, $\mathrm{H}$. \\
\hline Brown, I.A. & Goutard, F. & Naidu, A.S. & VanderZwaag, D. \\
\hline Brown, R.J. & Grenier, A.A. & Natcher, D.C. & Van West, P. \\
\hline Bruggeman, J. & Grimwood, B.S. & Noble, B. & Vermaire, J.C. \\
\hline Buckland, S.T. & Gunn, A. & Norton, P. & Verocai, G.G. \\
\hline Buonsanti, F. & Guy, E. & O’Hara, T.M. & Von Parijs, S. \\
\hline Burnett, K. & Haas, C. & O’Regan, M. & Voorhees, H. \\
\hline Cater, T. & Harwood, L.A. & Odess, D. & Vorano, N. \\
\hline Chamard, S. & Higdon, J.W. & Pearce, C. & Ween, G.B. \\
\hline Chételat, J. & Hobbs, W. & Pearce, T. & Wenzel, G.W. \\
\hline Chircop, A. & Holtgrieve, G. & Phillips, A.J. & Weslawski, J.M. \\
\hline Citta, J.J. & Hood, G.A. & Quakenbush, L.T. & Whitfield, D.P. \\
\hline Clark, D.A. & Howell, S.E.L. & Raitio, K. & Wilson, K. \\
\hline Craig, T. & Hudson, A. & Richard, J. & Wilson, S. \\
\hline Cyr, A. & Huntington, H.P. & Riseth, J.A. & Winegardner, A. \\
\hline Dannewitz, J. & Jätzold, R. & Robertson, G.J. & Wolfe, B.B. \\
\hline Dee, J. & Jenkins, E. & Roturier, S. & Wynne, M. \\
\hline DiNovelli, D. & Joly, K. & Roy-Léveillée, P. & Young, K.L. \\
\hline Dixon, B. & Jones, J. & Saladyga, $\mathrm{T}$. & Younie, A.M. \\
\hline Dodds, K. & Keatley, B. & Sandström, C. & \\
\hline Donoghue, H.D. & Keenleyside, A. & Schindler, D.E. & \\
\hline
\end{tabular}

